\title{
Boundary element model of the human head exposed to an electrostatic field generated by Video Display Units
}

\author{
D. Čavka ${ }^{1}$, D. Poljak ${ }^{1}$, A. Peratta ${ }^{2}$ \& C. Brebbia ${ }^{2}$ \\ ${ }^{1}$ University of Split, Croatia \\ ${ }^{2}$ Wessex Institute of Technology, UK
}

\begin{abstract}
The goal of this paper is to investigate the interaction between the electrostatic field of a Video Display Unit (VDU) and the human head located in front of it. Special attention is given to the field distribution over the surface of the face. The boundary element model for assessment of the electrostatic field, by solving Laplace equation for the electric potential, is implemented. The electrostatic field is calculated for two different faces and then compared to the results obtained by the other numerical methods. The advantage of the presented numerical method is a high resolution and a higher accuracy. The methodology development is equally applicable to other industrial electrostatic field problems.

Keywords: boundary element method, electrostatic field, human exposure, video display units.
\end{abstract}

\section{Introduction}

Video display units (VDUs) based on the cathode ray tube (CRT) are sources of several types of radiation e.g. $\mathrm{X}$ ray radiation, optical radiation (ultraviolet radiation, visible light and infrared radiation) electromagnetic radiation and electrostatic field. Along with the expanding use of VDUs some concerns about the effect of these fields on the human health have appeared. Over the years of work it has been noticed and proven that $\mathrm{X}$ ray radiation, optical radiation, high $(\sim \mathrm{MHz})$ and low $(\sim \mathrm{kHz})$ frequency electromagnetic fields are well below technical guidelines $[1,2]$ hence, considered not harmful for the health.

On the other hand, extremely low frequency (ELF) $(\sim \mathrm{Hz})$ electromagnetic and electrostatic fields might be associated with some skin diseases, suppression of melatonin, or induction of phosphenes in the eyes, despite the fact that there is no 
strong evidence of adverse health effects from domestic levels of ELF electromagnetic fields [1-3].

The present work deals with electrostatic fields, as large electrostatic fields have been detected from several types of VDUs and possibly related with skin rashes $[3,4]$. Moreover, regarding health effects, the electrostatic fields are still neither fully investigated nor completely understood. To the best of authors' knowledge, no standards or guidelines exist for electrostatic fields. However, it has to be pointed out that the main concern due to exposure to these fields is linked with particle transport and deposition [5].

In the present paper, the realistic, three-dimensional, anatomically based model of the human head exposed to electrostatic field from VDU is presented. The electrostatic field around human head is assessed using the Boundary Element Method (BEM) [6, 7]. Special attention is given to the shape of the face by investigating two different faces. Furthermore, the field strength on the nose tip and eyes has been studied. Also, results obtained within this work are compared to those found by different methods, i.e. Finite Differences (FDM) [3] and Finite Elements (FEM) [8]. The principal motivation for studying this problem is lack of information of electrostatic field at the surface of human face, and the need to investigate accuracy of the results already obtained and published. Such information then can be used for solving problems like particle deposition on human face and eyes.

Another aim of the present work is to demonstrate that BEM is ideally suited to modelling of complex surfaces such as the human face, and that the results obtained are highly accurate.

\section{Formulation of the problem}

Assuming the charge density to be negligible, the mathematical description of the 3D electrostatic field between a VDU and human head is given by Laplace equation for electric potential $\varphi$ :

$$
\nabla^{2} \varphi=0
$$

with the associated boundary conditions:

$$
\begin{gathered}
\varphi=\varphi_{s} \text { on the display, } \\
\varphi=\varphi_{h} \text { on the head, } \\
\nabla \varphi \cdot \vec{n}=0 \text { on the far field boundaries. }
\end{gathered}
$$

As shown in figure 1, the Dirichlet boundary conditions (2) and (3) are specified on the face and the display, while Neumann conditions (4) are imposed on all remaining exterior boundaries.

It is worth noting that the head is considered to be the perfect conductor thus being itself an equipotential surface with potential $\varphi_{h}$. 


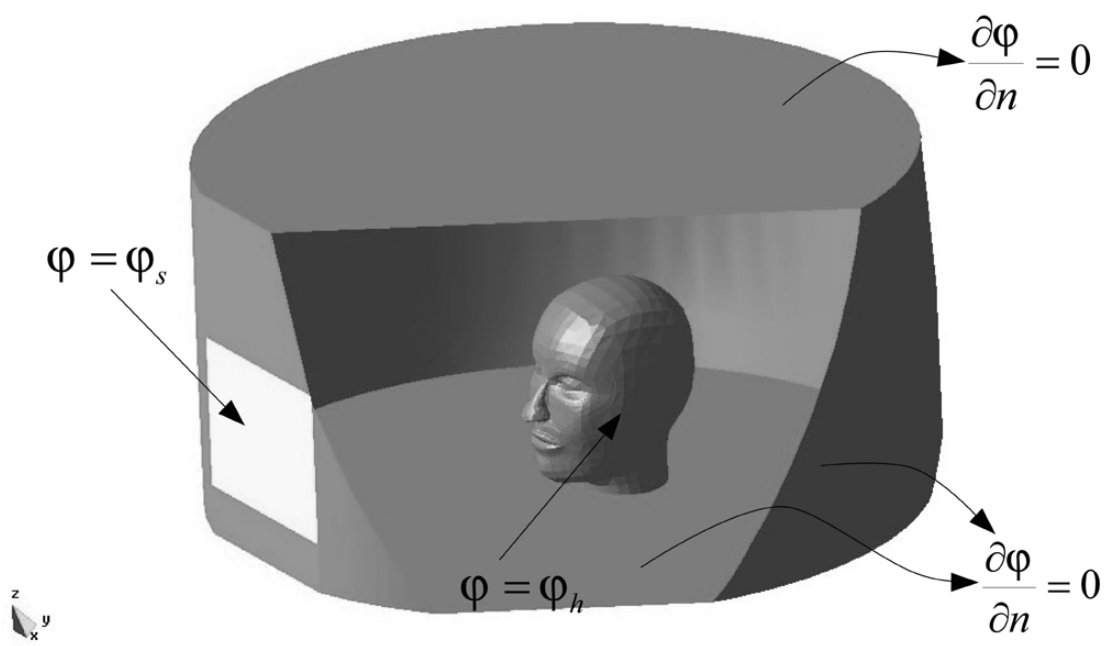

Figure 1: Geometry and boundary conditions for numerical 3D model of human seated in front of a VDU.

\section{Model parameters and numerical solution}

\subsection{Model properties}

Presented formulation involves the parameters $l_{s}, d_{s}, \varphi_{s}$ and $\varphi_{h}$. They represent distance between display and nose tip, size of display (diagonal display size given in inches), electrostatic potential on display and electrostatic potential on head, respectively. The mean electric potential on a CRT monitors is in the range of $1-15 \mathrm{kV}$. In this case the electric potential of the display is assumed to be relatively very high $(15 \mathrm{kV})$ and it is considered as approximately worst-case scenario. In the present work standard conditions for the person in front of display are defined in table 1.

Table 1: $\quad$ Standard conditions.

\begin{tabular}{|l|c|c|c|c|}
\hline Magnitude & $l_{s}(\mathrm{~m})$ & $d_{s}(\mathrm{in})$ & $\varphi_{s}(\mathrm{kV})$ & $\varphi_{h}(\mathrm{kV})$ \\
\hline Value & 0.4 & 17 & 15 & 0 \\
\hline
\end{tabular}

It is worth emphasizing that, the eyebrows are considered to have the same potential as the face. Other parameters such as temperature, humidity and conductivity of the surface of the screen glass are not considered in order to simplify the model. But, it has to be underlined that air humidity is very important factor, and in this calculations is considered very low (dry air).

Attention is given to shape of the face by investigating two different face geometries: person 1 (Figure 2 a)) and person 2 (Figure 2 b)). 
The geometrical information of the models was introduced by means of the software 3D Studio Max, while the computational domain was preprocessed in GiD [9]. GiD is commercial software for professional pre and post processing developed by International Center for Numerical Method in Engineering, Barcelona, Spain. The postprocessing was performed using GiD, as well.

\subsection{Boundary element solution}

Applying the weighted residual approach, equation (1) can be integrated over the calculation domain $\Omega$ :

$$
\int_{\Omega} \nabla^{2} \varphi \psi d \Omega=0
$$

where $\psi$ is the weighting function. Using the following identities:

$$
\begin{aligned}
& \nabla \cdot(\nabla \varphi \psi)=\psi \nabla^{2} \varphi+\nabla \varphi \cdot \nabla \psi \\
& \nabla \cdot(\varphi \nabla \psi)=\nabla \varphi \cdot \nabla \psi+\varphi \nabla^{2} \psi
\end{aligned}
$$

and applying the generalised Gauss theorem equation (5) can be written, as follows:

$$
\int_{\Omega} \psi \nabla^{2} \varphi d \Omega=\int_{\Gamma} \psi \frac{\partial \varphi}{\partial n} d \Gamma-\int_{\Gamma} \varphi \frac{\partial \psi}{\partial n} d \Gamma+\int_{\Omega} \varphi \nabla^{2} \psi d \Omega
$$

The weighting function $\psi$ can be chosen to be the solution of the differential equation, i.e.

$$
\nabla^{2} \psi-\delta\left(\vec{r}-\vec{r}^{\prime}\right)=0
$$

where $\delta$ is the Dirac delta function, $r$ denotes the observation points and $r$ ' denotes the source points. The solution of equation (9) is called the fundamental solution or Green function.

Thus, the domain integral in (8) can be written:

$$
\int_{\Omega} \varphi \nabla^{2} \psi d \Omega=-\int_{\Omega} \varphi \delta\left(\vec{r}-\vec{r}^{\prime}\right) d \Omega=-\varphi_{i}
$$

for any point inside the domain.

Combining equations (8)-(10) the following integral relation is obtained:

$$
\varphi_{i}=\int_{\Gamma} \psi \frac{\partial \varphi}{\partial n} d \Gamma-\int_{\Gamma} \varphi \frac{\partial \psi}{\partial n} d \Gamma
$$

The integral equation (11) can be regarded as the Green representation of the function $\varphi$.

The function $\psi$ is the fundamental solution of the equation (9). For threedimensional problems it is given by the expression:

$$
\psi=\frac{1}{4 \pi R}
$$


where $R=\left|\vec{r}-\vec{r}^{\prime}\right|$ is the distance from the source point (boundary point) to the observation point.

When the observation point $i$ is located on the boundary $\Gamma$, the boundary integral becomes singular as $R$ approaches zero. In order to deal with this singularity a small sphere for $3 \mathrm{D}$ problems is considered.

Calculating the surface integrals over the small spheres around singular points, finally follows the integral formulation of Laplace equation (1):

$$
c_{i} \varphi_{i}=\int_{\Gamma} \psi \frac{\partial \varphi}{\partial n} d \Gamma-\int_{\Gamma} \varphi \frac{\partial \psi}{\partial n} d \Gamma
$$

where

$$
c_{i}=\left\{\begin{array}{l}
1, \quad i \in \Omega \\
1 / 2, \quad i \in \Gamma \text { (smooth boundary) }
\end{array}\right.
$$

Having computed the scalar potential distribution the electrostatic field can be determined from expression:

$$
\vec{E}=-\nabla \varphi
$$

\subsection{Boundary discretisation}

The three terms in equation (13) involve only boundary integrals. The boundary $\Gamma$ can be discretised into $N_{e}$ boundary elements, so that

$$
\begin{gathered}
\Gamma=\sum_{j=1}^{N_{e}} \Gamma_{j} \\
c_{i} \varphi_{i}=\sum_{j=1}^{N_{e}} \int_{\Gamma_{j}} \psi \frac{\partial \varphi}{\partial n} d \Gamma-\sum_{j=1}^{N_{e}} \int_{\Gamma_{j}} \varphi \frac{\partial \psi}{\partial n} d \Gamma
\end{gathered}
$$

Each boundary element $j$ contains a number $\left(N_{j}\right)$ of collocation nodes, in which the potential or fluxes are evaluated. Thus, the values of the potential or its normal derivative at any point defined by the local coordinates $\left(\xi_{1}, \xi_{2}\right)$ on a given boundary element can be defined in terms of their values at the collocation nodes, and the $N_{j}$ interpolation functions $\Phi_{k}$ with $k=1, \ldots, N_{j}$ as follows:

$$
\begin{gathered}
\varphi(\xi)=\sum_{k=1}^{N_{j}} \Phi_{k}(\xi) \varphi_{k} \\
\frac{\partial \varphi(\xi)}{\partial n}=\sum_{k=1}^{N_{j}} \Phi_{k}(\xi) \frac{\partial \varphi_{k}}{\partial n}
\end{gathered}
$$

Discretising the boundary and applying the collocation technique, expression (8) can be rewritten in the following form: 


$$
c_{i} \varphi_{i}=\sum_{j=1}^{N_{e}} \sum_{k=1}^{N_{j}}\left(\int_{\Gamma_{j}} \psi \Phi_{k} d \Gamma_{j}\right) \frac{\partial \varphi_{j k}}{\partial n}-\sum_{j=1}^{N_{e}} \sum_{k=1}^{N_{j}}\left(\int_{\Gamma_{j}} \frac{\partial \psi}{\partial n} \Phi_{k} d \Gamma_{j}\right) \varphi_{j k}
$$

The notation in (20) can be simplified by making the use of matrix notation:

$$
H[\varphi]=G\left[\frac{\partial \varphi}{\partial n}\right]
$$

where

$$
\begin{gathered}
H_{i l}=\delta_{i l} c_{i}+\int_{\Gamma_{j}} \frac{\partial \psi_{i}}{\partial n_{j}} \Phi_{k} d \Gamma_{j} \\
G_{i l}=\int_{\Gamma_{j}} \psi_{i} \Phi_{k} d \Gamma_{j}
\end{gathered}
$$

where index $l$, identifying a collocation node within the domain, can be calculated in terms of indices $j$ and $k$, by means of the nodal connectivity of the mesh, i.e. index $l=1, \ldots, M$. and $M$, is the total number of collocation nodes in the domain. Basically, the index $l$ is used to identify one of the adjacent freedom (collocation) nodes from a global point of view, and is given as a function of the indicator of element $(j)$, and the local collocation node of that element $(k)$. In the case of discontinuous collocation nodes:

$$
M=\sum_{j=1}^{N_{e}} N_{j}
$$

The boundary differential $d \Gamma_{j}$ can be expressed in terms of the domain local coordinates $(\xi)$ via the jacobian of the transformation $|J|$ :

$$
d \Gamma_{j}=|J| d \xi_{1} d \xi_{2}
$$

Finally, equation (21) can be written as a given vector in function of the source term, or a characteristic matrix in function of the unknown potentials and normal fluxes at the collocation nodes of the boundary, the application of the prescribed boundary conditions and the assembly of the linear set of equations, that (25) produces, yields to a determined system of equations of dimensions $N b y \quad N$ of the form:

$$
\mathbf{A} \mathbf{x}=\mathbf{b}
$$

where the vector of unknowns $\mathbf{x}$ contains the potentials and normal fluxes that were not prescribed as boundary conditions, the matrix $A$ involves the coefficients of $H$ and $\mathrm{G}$, and the right hand side term involves the source term, and the boundary conditions.

\section{Results and discussion}

The mesh generated for the model of person 1 contains around 4500 constant elements, while for the model of person 2 around 6000 (figure 2). The difference 
is due to number of surfaces that form particular head. Numerical calculation was carried out using MATLAB code.

Figure 3 shows the electrostatic field $E[\mathrm{~V} / \mathrm{cm}]$ on the face of person 1 and person 2.

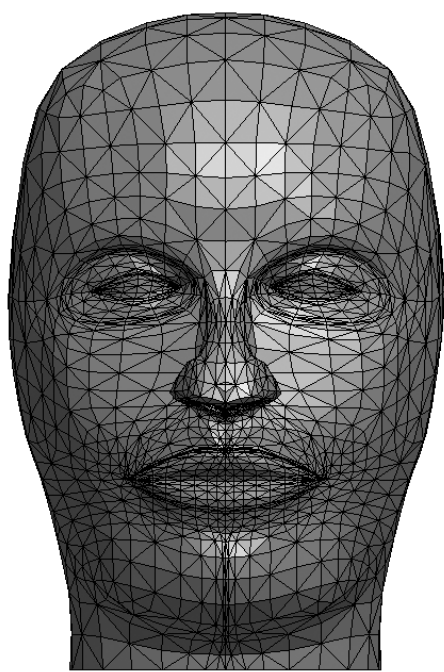

a)

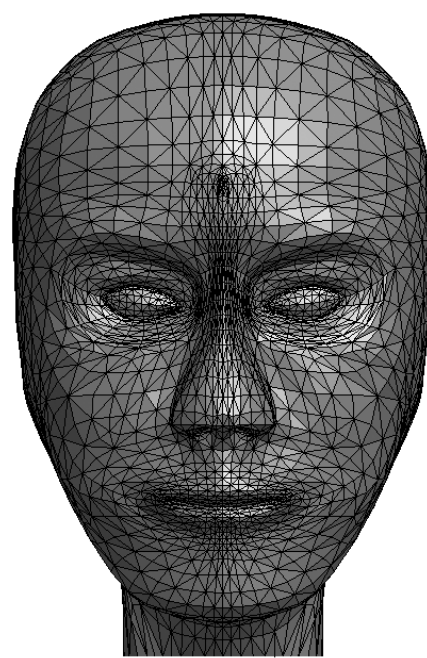

b)

Figure 2: $\quad$ BEM discretisation of the heads: a) person 1; b) person 2.
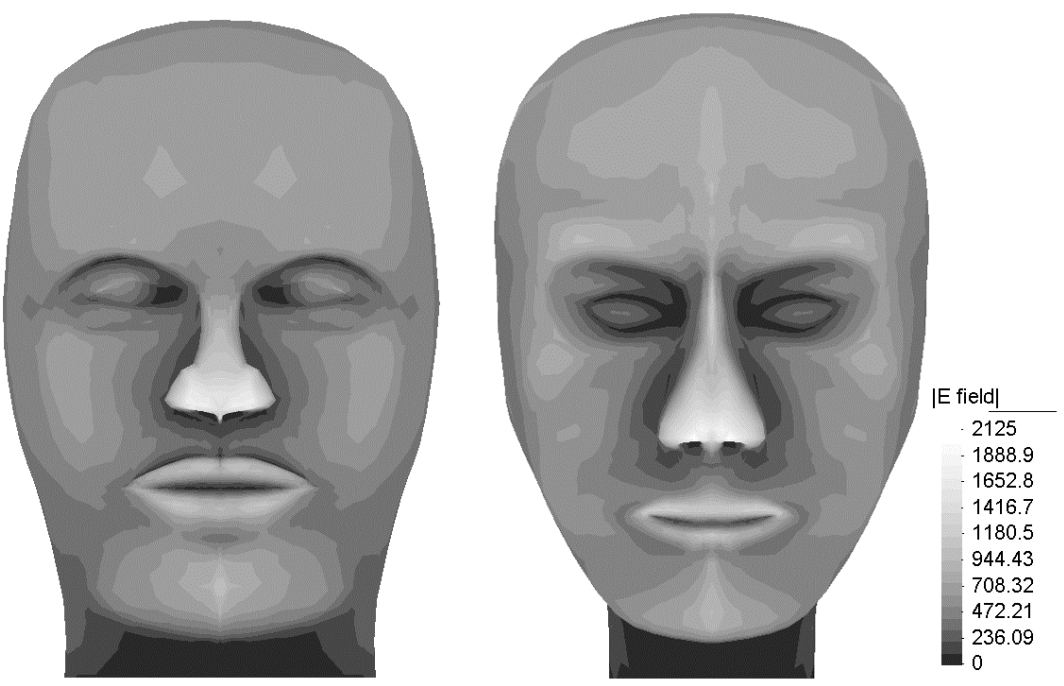

Figure 3: Electrostatic field strength on the face of persons. 
The results show very different values of field strength in the two cases, i.e. the maximal value of the field at the nose of the person $1(1760 \mathrm{~V} / \mathrm{cm}=176$ $\mathrm{kV} / \mathrm{m})$ is lower then the value for the person $2(2125 \mathrm{~V} / \mathrm{cm})$. The difference is caused by the different shape of the face, particularly the shape of the nose. For most of the face area, the difference is not so significant. Certain difference is noticed on the forehead and brows, around $300 \mathrm{~V} / \mathrm{cm}$. Relatively high values, compared to the rest of the face, of electrostatic field can be found on the lips, chin and on the top of the cheek, around $1000 \mathrm{~V} / \mathrm{cm}$.

These details and differences are clearly visible from the perspective view of faces around nose and eyes, as shown in figure 4 .
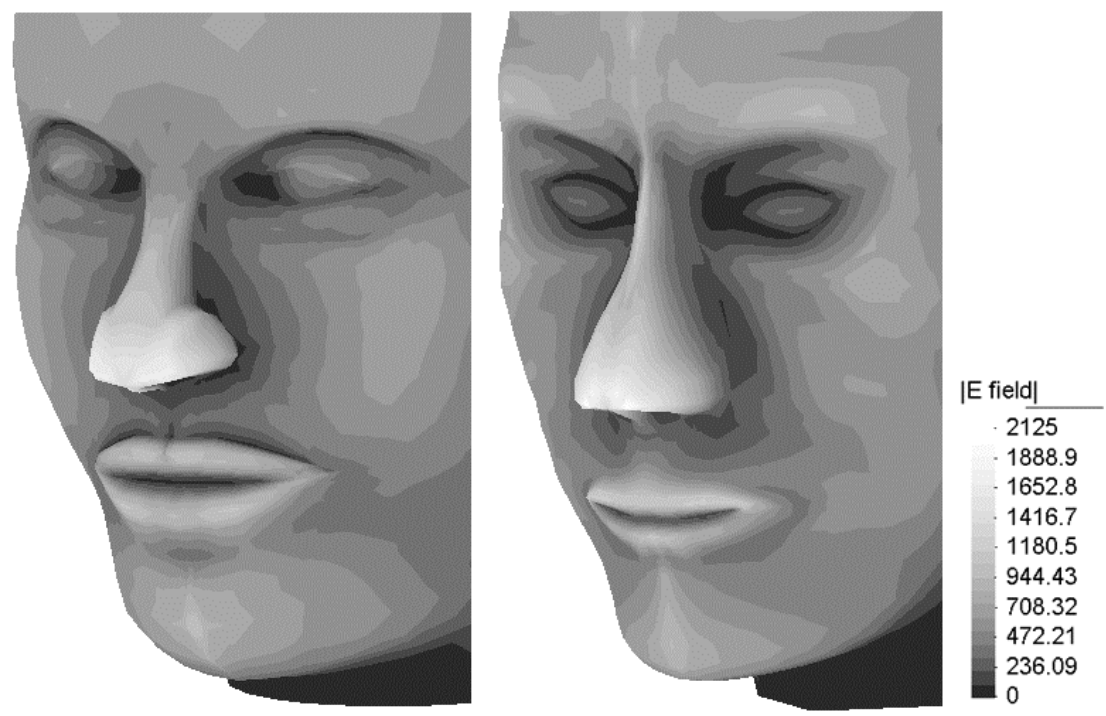

Figure 4: Electrostatic field strength $[\mathrm{V} / \mathrm{cm}]$ around the nose and eyes.

It can be observed from figure 4 that the field strength is highly dependent on the shape of the face especially of the nose. Also from the same figure is clear that field strength around eyes is relatively low comparing to the rest of the face, around 400-500 V/cm.

Results obtained in this work are comparable with the ones obtained by different methods and presented in earlier publications such as [3] (FDM) and [8] (FEM). Exact comparison with results obtained in [3] cannot be made due to different shapes of the heads being considered. What should be stated is that the values of electrostatic field are very similar and the behaviour of the field on the face is similar in nature. Comparing results obtained in this work with the results obtained in [8] using FEM for the same head models some differences arise.

On the FEM model some "hot spots" (i.e. regions of high electrostatic field strength) can be noticed on the nose, having much higher values of the field than the same spots on the BEM model. Although, there are other parts where the values of the BEM are higher than those of the FEM. 
Nevertheless, overall both methods give very similar results. The differences can be explained because of the difficulty of defining sufficiently refined FEM meshes around complex geometries such as the human face.

\section{Conclusions}

This paper investigates the interaction between the electrostatic field generated by VDUs and the human head.

As different from previous studies using Finite Differences (FDM) or Finite Elements (FEM), this work applies the Boundary Element Method (BEM), which generally is more accurate and versatile than the other two, allowing for a better representation of the shape of the human face.

Two different facial shapes were investigated and results compared with those obtained by FEM. Although both results were of similar accuracy the BEM allows for a better description of the human face.

Future work will include other factors such as air humidity and more refined models for area of complex geometry such as the ears. The mesh will also be refined in the region around the eyes introducing eyelashes and eyebrows. The work presented in this paper is important not only to study the electrostatic field effects of VDU but also of a whole range of other industrial equipments.

\section{References}

[1] International Commission on Non-Ionizing Radiation Protection (ICNIRP) and International labour organization: "Visual display units: Radiation protection guidelines", International labour office, Geneva 1994.

[2] Institute of Electrical and Electronics Engineers, Biological and health effects of electric and magnetic fields from video display terminals, IEEE Engineering in Medicine and Biology Magazine 16(3): 87-92, 1997.

[3] Nielsen, N.F., Michelsen, J., Michalsen, J.A.. \& T. Schneider, Numerical calculation of electrostatic field surrounding a human head in visual display environments", Journal of electrostatic, 36, pp. 209-223, 1996.

[4] Australian Radiation Protection and Nuclear Safety Agency, Radiation Emission from Video Display Terminals, Australian Radiation Protection and Nuclear Safety Agency, 2003.

[5] Nielsen, N.F. \& Schneider, T., Particle Deposition onto a Human Head: Influence of Electrostatic and Wind Fields, Bioelectromagnetics 19, pp. 246-258, 1998.

[6] Brebbia, C.A. \& Dominguez, J., Boundary Elements, An Introductory Course, second edition. WIT Press: Boston, Southampton 1998.

[7] Gonzalez, C., Peratta, A. \& Poljak, D., Boundary Element Modelling of the Realistic Human Body Exposed to Extremely-Low-frequency (ELF) electric Fields: Computational and Geometrical Aspects, IEEE Transactions on Electromagnetic Compatibility, Vol. 49, No 1 pp. 153-162, February 2007. 
124 Boundary Elements and Other Mesh Reduction Methods XXX

[8] Čavka, D., Poljak, D. \& Peratta, A., Finite element model of the human head exposed to electrostatic field generated by Video Display Units, SoftCOM conference proceedings, Split 2006.

[9] GiD 7 Reference Manual. International Center for Numerical Methods in Engineering. CIMNE. Spain. 2006. (http://gid.cimne.upc.es/). 